International Journal of Psychological Research and Reviews
(ISSN:2639-6041)

\title{
Touching and Touch Deprivation During a COVID-19 Lockdown
}

\author{
Tiffany Field ${ }^{1,2}$, Samantha Poling ${ }^{2}$, Shantay Mines ${ }^{2}$, Debra Bendell ${ }^{2}$, and Connie Veazey ${ }^{2}$ \\ ${ }^{1}$ University of Miami/Miller School of Medicine \\ ${ }^{2}$ Fielding Graduate University
}

\section{ABSTRACT}

Touching your kids and your partner, self-touching, and touch deprivation have had different effects on individuals during a COVID-19 lockdown. In this Survey Monkey study conducted during a COVID-19 lockdown ( $\mathrm{N}=260$ respondents), 26\% said they were touch deprived a lot, $21 \%$ said they were touching their kids a lot, $33 \%$ touching their partner a lot, and 32\% self-touching a lot (e.g. yoga and stretching). Correlation analyses suggested that touch deprivation was related to scores on the Stress, Anxiety, Depression, Fatigue, Sleep Disturbances and PTSD scales. The three types of touching were positively related to scores on the Health Scale, at home projects, and outdoor exercising with others. Touching partner was also related to lower scores on the Stress, Depression, and PTSD Scales and Self-touching was related to lower scores on the Fatigue and Sleep Disturbance Subscales. The results of these data analyses are limited by the self-reported data from a non-representative, cross-sectional sample. Nonetheless, they highlight the negative effects of touch deprivation and the positive effects of touching your kids and partners and self-touch during a COVID-19 lockdown.

Keywords: health, stress, touch deprivation, touching your kids, touching partners
*Correspondence to Author:

Tiffany Field1,2

${ }^{1}$ University of Miami/Miller School of Medicine; ${ }^{2}$ Fielding Graduate University

How to cite this article:

Tiffany Field, Samantha Poling, Shantay Mines, Debra Bendell, Connie Veazey. Touching and Touch Deprivation During a COVID-19 Lockdown. International Journal of Psychological Research and Reviews, 2020, 3:42.

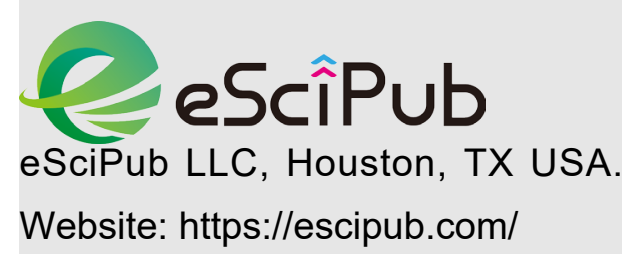


Stress, anxiety, depression, fatigue, sleep disturbances and PTSD symptoms have been reported in several studies on COVID-19 lockdowns. ${ }^{1-6}$ Despite the likelihood that touch deprivation also occurs during lockdowns, the words touch deprivation, touch, and touching could not be found in the extensive COVID-19 lockdown literature. That was surprising given that dozens of social media pieces (newspaper and magazine articles and radio shows) have focused on touch deprivation occurring during the lockdowns and its impact on mental and physical health.

Finding studies on touch deprivation in the literature was difficult inasmuch as touch deprivation effects have been almost exclusively studied in extreme touch deprivation situations such as Romanian orphanages. An exception was a study on adolescents suggesting that those who received less touching showed more verbal and physical aggression. ${ }^{7}$ Studies on touching are more common than research on touch deprivation. In pre-COVID-19 studies on touching, behavioral and physiological responses to touching someone and being touched by someone have typically been positive. ${ }^{8}$ Most of those studies took place in laboratory settings where touching was noted to have positive effects including decreased stress when couples hugged each other or held hands prior to and during stressful conditions like giving a speech or solving math problems. ${ }^{9}$ Anxiety and depression were decreased and physical health was improved by touching in several of those studies. ${ }^{10}$ By decreasing anxiety and depression and the associated stress hormones, e.g. cortisol, immune function has been improved. This was illustrated, for example, in a study showing that hugging was associated with lower cortisol and better immune function. ${ }^{11}$

Very few researchers have studied touching during natural stressors. An exception was a pre-COVID naturalistic observation study that took place at airport gate waiting areas. ${ }^{12} \mathrm{~A}$ preliminary analysis of those data suggested that a form of touch deprivation was occurring even before COVID-19 lockdowns, as evidenced by fellow travelers being on cell phones $68 \%$ of their waiting time. They were scrolling and texting, not touching.

The purpose of the present data analyses was to determine the prevalence of different types of touching that naturally occurred during a COVID-19 lockdown based on survey data and whether touching ameliorated the feelings of touch deprivation as well as the other stressors associated with lockdowns. In a previous analysis of these survey data, touch deprivation was lessened by exercise. ${ }^{12}$ Whether touching kids and partners/friends could alleviate touch deprivation was addressed in the current data analyses. The other questions addressed in the current data analyses were the relationships between touching your partner, touching your kids, and self-touch. The data were entered into correlation analyses to determine the relationships between the different forms of touch and the associations between the different types of touch and lockdown-related stressors. The stressors were assessed on a scale created for the survey as well as standardized scales on anxiety, depression, sleep disturbances, fatigue, and PTSD symptoms. In addition, relationships between the different forms of touch and other activities were assessed including healthy behaviors, at home projects, social media, and communication with others.

Because the majority of the participants were expected to be living with families and would have increased time with each other during a lockdown, touching partners and touching kids were expected to be prevalent activities. In addition, the different forms of touch were expected to be positively related to each other and negatively related to feeling touch deprived. Further, these forms of touching were expected to be related to other positive activities including healthy behaviors, at home projects, and connecting with others and negatively related to the COVID-19 lockdown related problems in the literature including stress, anxiety, depression, 
sleep disturbances, fatigue and PTSD symptoms.

\section{Methods}

\section{Participants}

A G* power analysis indicated that a sample size of 224 was required for an alpha of .05 and $80 \%$ power. The participants included individuals $(\mathrm{N}=260)$ who ranged in age from 18-82 $(\mathrm{M}=47$ years). Gender was distributed $79 \%$ female, $18 \%$ male and $3 \%$ other (non-specified). Ethnicity was distributed 68\% Non-Hispanic White, $21 \%$ Hispanic, 3\% Black and $8 \%$ other (non-specified). Professions were distributed $35 \%$ office worker, $30 \%$ academic, $15 \%$ managerial, $12 \%$ medical and $8 \%$ labor. The average income was $\$ 72,572,28 \%$ were unemployed and $69 \%$ worked at home. Twentythree per cent lived alone.

\section{Procedure}

A flyer was posted on Facebook giving a brief description of the study including some sample items and the age criterion being greater than 18 years. The Facebook flyer included a link to the survey on Survey Monkey which included 11 scales for a total of 87 items. The survey was four weeks duration (April 1-30, 2020), and the data were directly transported to SPSS for data analyses.

\section{Measures}

The survey included several demographic items including those already mentioned (age, gender, ethnicity, profession, income, type of employment, working at home, and living alone). The following five scales were created specifically for this survey to relate to activities and stress associated with the COVID-19 lockdown. ${ }^{13}$ The participants rated the items on the scales from zero meaning "not at all" to three meaning "a lot" including the:

1) Health Scale (15 items) (Cronbach's alpha=.66) which included exercise (inside exercise, outside exercise, and outside exercise with others as well as the types of exercise like walking and running), touching (touching partner, touching kids, and touching self, e.g. brushing in shower, yoga, and stretching as well as the types of touching like hugging and backrubs), COVID- 19-related safety practices including washing hands and social distancing, self-care, spiritual activities (meditating and feeling spiritual), and liking being at home. A factor analysis yielded three factors contributing to $47 \%$ of the variance on the Health Scale score: Factor 1 "Self/Spiritual Care" included Meditating (.74), Self-Care (.68), and Feeling Spiritual (.77) items that together explained $23 \%$ of the variance; Factor 2 "Touching" included the items Touching your kids (.75) and Touching your partner or friend (.72) that explained $14 \%$ of the variance; and Factor 3 "Exercise" included the items Outside exercise (-.89) and Exercise outside with someone else $(-.76)$ that explained $10 \%$ of the variance;

2) Media/Communications Scale (10 items) (Cronbach's alpha $=.58$ ) including talking on the phone, texting, on Internet, gaming, Facebook/Instagram time, receiving and sending messages/media about the virus, engaging in Zoom/Skype/Facetime activities (e.g. Yoga, meditation), watching the news, watching other TV programs, and watching movies. A factor analysis yielded four factors contributing to $61 \%$ of the variance on the Media/Communication Scale score: Factor 1 "Entertainment" included the items Watching movies (.84) and TV programs (.80) that explained $23 \%$ of the variance; Factor 2 "Communication" included phone use (.80), texting (.70) and Zoom (.63) that explained 14\% of the variance; Factor 3 "Social Media" included being on internet (.78) and Facebook time (.60) that explained $13 \%$ of the variance; and Factor 4-"COVID News" that included watching the news (.79) and messaging about the virus (.60) that explained $11 \%$ of the variance;

3) Connecting Scale (4 items) (Cronbach's alpha=.41) which included connecting with friends, trying to connect with old friends, helping children do homework, and receiving support from others; 
4) Working Scale (6 items) (Cronbach's alpha=.61) including cooking, caregiving, housekeeping, paperwork, creative work, and working on projects/hobbies; and

5) Stress Scale (11 items) (Cronbach's alpha=.78) which included worrying about getting a virus, worrying about your financial status, wanting this experience to end, feeling isolated, feeling lonely, feeling bored, feeling touch deprived, snacking, drinking alcohol, napping, and getting "cabin fever". A factor analysis yielded three factors contributing to 56 $\%$ of the variance on the Stress Scale score: Factor 1 "Stimulation deprivation" included the items Feeling Isolated (.86), Feeling lonely (.86), Feeling bored (.74), Getting cabin fever (.70), and Feeling touch deprived (.65) that together explained $34 \%$ of the variance; Factor 2" Worrying" included the items Worried about finances (.67) and Worried about the virus (.47) explained $12 \%$ of the variance; and Factor 3 "Stress behaviors" included the items Napping (.68) and Snacking (.53) that explained $10 \%$ of the variance.

The standardized scales on the survey included 4 PROMIS Subscales ${ }^{14}$ (each item was rated on a 5 -point scale as $1=$ never, $2=$ rarely, $3=$ sometimes, $4=$ often, and $5=$ always) which included the: 1) PROMIS Anxiety Subscale (4 items) (Cronbach's alpha=.88) which included I felt fearful, I found it hard to focus on anything other than my anxiety, my worries overwhelmed me, and I felt uneasy;

2) PROMIS Depression Subscale (4 items) (Cronbach's alpha=.91) that included I felt worthless, helpless, depressed, and hopeless;

3) PROMIS Fatigue Subscale (3 items) (Cronbach's alpha=.92) including I felt fatigued, I had trouble starting things because I'm tired, and I felt run-down; and

4) PROMIS Sleep Disturbance Subscale (4 items) (Cronbach's alpha $=.86$ ) that included my sleep quality was bad, my sleep is not refreshing, I had a problem with my sleep, and I had difficulty falling asleep.
The second standardized scale was a PTSD Screener entitled "PTSD-8: A short PTSD Inventory" (8 items) (Cronbach's alpha $=.92) .{ }^{15}$ This inventory is introduced by the statement "If you're being reminded of a traumatic experience, please rate how much the following have bothered you during the lockdown" as: 0 ) not at all, 1) rarely, 2) sometimes, and 3) most of the time. The items are: recurrent thoughts and memories of the event, feeling as though the event is happening again, recurrent nightmares about the event, sudden emotional or physical reactions when reminded of the event, avoiding activities that remind you of the event, avoiding thoughts or feelings associated with the event, feeling jumpy/easily startled, and feeling on guard.

\section{Results}

\section{Correlation Analyses Yielding Significant Coefficients for Touch Deprivation}

Results indicated that $60 \%$ of the sample reported being touch deprived (0 (39\%) not at all, $1(19 \%), 2(16 \%), 3(26 \%)$ a lot. Correlation analyses revealed a number of significant correlation coefficients for touch deprivation(at the $p<.05$ level with most at the $p=.0001$ level) including the demographic variable of more often "living alone", and the following (see Table 1 for the correlation coefficients for the scales total scores):1) for the Health Scale total score, and the items including less exercise with others, less touching partner, less touching your kids, less liking being home, and less self-care; 2) for items on the Media/Communication Scale including more texting, more Zoom, and less watching movies; 3 ) less Connecting with friends; 4) on the Working Scale for the items including less homework with kids, less cooking, housework, and paper work; 5) for the total score on the Stress Scale and for its items including greater worrying about the virus, wanting this experience to end, feeling isolated, lonely, and bored and greater alcohol, napping, and cabin fever; 6) for the PROMIS Anxiety Subscale total score and all its items (feeling fearful, focus on anxiety, overwhelming worries, and feeling 
uneasy); 7) for the total score on the PROMIS Depression Subscale, and all its items including feeling worthless, helpless, depressed, and hopeless; 8) for the total score on the PROMIS Fatigue Subscale, and all its items including fatigue, tired, and run-down; 9) for the total score on the PROMIS Sleep Disturbance Subscale, and all its items including quality of sleep, refreshing sleep, problems with sleep, and falling asleep; and 10) for the total score on the PTSD-8 Inventory and all its items including recurrent thoughts or memories, feeling the event is happening again, recurrent nightmares, sudden emotional and physical reactions, avoiding activities that remind you of the event as well as thoughts and feelings associated with the event, feeling jumpy/easily startled and feeling on guard.

Table 1. Correlation coefficients for significant relationships between touch deprived ratings and scores on COVID-19 Lockdown Activities Survey scales and subscales.

\begin{tabular}{lcr}
\hline Measure & Correlation coefficient & p level \\
\hline Health Scale Score & -.28 & .000 \\
Stress Scale Score & .64 & .000 \\
PROMIS Anxiety Subscale Score & .39 & .000 \\
PROMIS Depression Subscale Score & .43 & .000 \\
PROMIS Fatigue Subscale Score & .30 & .000 \\
PROMIS Sleep Disturbance Subscale Score & .27 & .000 \\
Posttraumatic Stress Inventory Score & .35 & .000 \\
\hline
\end{tabular}

Table 2. Correlation coefficients for significant relationships between touching your kids and scores on COVID-19 Lockdown Activities Survey scales and subscales.

Measure Correlation coefficient p level

Health Scale Score

Connecting Scale Score

\section{Correlation Analyses Yielding Significant} Coefficients for Touching Your Kids

Results indicated that $38 \%$ of the sample reported touching your kids (rated $0(62 \%)$ not at all, $1(8 \%), 2(9 \%), 3(21 \%)$ a lot). The primary types of touching your kids that were given by the participants included hugging (52\%), kissing $(13 \%)$, backrubs (7\%), and cuddling (6\%)..Correlation analyses revealed a number of significant correlations (at least at the $p<.05$ level) between touching your kids and several demographic variables including being a younger age, being employed, having more schooling, not living alone, having more family at home and having more kids living with you. Significant correlation coefficients were also noted for the following scales and items on scales (see Table 2 for the correlation coefficients for the scales' total scores):1) a positive correlation for the Health Scale total score and for its items including more outdoor exercise with someone else, more touching partner, and more washing hands but less selfcare; 2) positive correlations for the Media/Communication Scale items indicating that touching your kids was related to more use of Facebook and more watching movies; 3) a positive correlation with the total score on the Connecting Scale and its item connecting with IJPRR:https://escipub.com/international-journal-of-psychological-research-and-reviews/ 
friends and helping children with homework; 4) a positive correlation with the Working Scale total score and its items including more caregiving, more housekeeping, and more paperwork; 5) correlations with Stress Scale items including feeling less touch deprived, more snacking, and less napping; 6) a negative correlation with the Sleep Disturbance Subscale item indicating less trouble falling asleep; and 7) a negative correlation for PTSD-8 scale items including being less reminded of other trauma and less avoiding the thoughts of other trauma.

\section{Correlation Analyses Yielding Significant Coefficients for Touching Your Partner}

Results indicated that $63 \%$ of the sample reported touching your partner (rated $0(37 \%)$ not at all, $1(10 \%), 2(20 \%), 3(33 \%)$ a lot). The primary types of touching your partner that were given by participants included hugging (39\%), kissing (19\%), backrubs (9\%), and cuddling (7\%). Correlation analyses revealed a number of significant correlation coefficients for touching your partner (at least at the $p<.05$ level) and demographic variables including being a younger age, less living alone, and having more kids living with you. Touching your partner was also significantly correlated with total scores on the scales and scale items including the following (see Table 3 for the correlation coefficients for the scales' total scores): 1) a positive correlation for the Health Scale Total Score and its items including more outdoor exercise with someone else and more selftouching (e.g. stretching, brushing in shower, and yoga); 2) a positive correlation on Media/Communication Scale items indicating more watching tv and movies; 3) a positive correlation with the Connecting Scale item helping children with homework; 4) a positive correlation with the Working total score and its items suggesting more cooking, caregiving and housekeeping; 5) a negative correlation with the Stress Scale total score and its items suggesting being less lonely and less touch deprived; 6) a negative correlation with the PROMIS Depression Subscale total score and its items feeling worthless, depressed, and hopeless; 7) a negative correlation with the PROMIS Fatigue Subscale total scale score item indicating feeling less tired; and 8) negative correlations with PTSD-8 Scale items including being less reminded of other trauma and feeling less on guard.

Table 3. Correlation coefficients for significant relationships between touching your partner and scores on COVID-19 Lockdown Activities Survey scales and subscales.

\begin{tabular}{lcc}
\hline Measure & Correlation coefficient & p level \\
\hline Health Scale Score & .48 & .001 \\
Working Scale Score & .25 & .01 \\
Stress Scale Score & -.16 & .05 \\
PROMIS Depression Subscale Score & -.19 & .01 \\
PTSD-8 Inventory Score & -.22 & .005 \\
\hline
\end{tabular}

Correlation Analyses Yielding Significant Coefficients for Self-Touching

Results indicated that $91 \%$ of the sample reported self-touching (rated $0(9 \%)$ none, 1 $(23 \%), 2(35 \%), 3(33 \%)$ a lot). The primary types of self-touching that were reported by the participants included stretching (36\%), brushing self in the shower (33\%), and yoga (24\%). The correlation analyses revealed a significant correlation between self-touching and gender indicating that more women reported selftouching. Significant correlations also occurred for total scale scores and their items (at least at the $p<.05$ level) (see Table 4 for the correlation 
coefficients for the scales' total scores) including: 1) a positive correlation for the Health Scale total score and its items indicating more exercise of each kind (indoor, outdoor, and outdoor exercise with someone else), more touching partner, and more self-care; 2) positive correlations on the Media/Communication Scale items including more phoning, more texting, and more time on zoom; 3) positive correlations with the Connecting Scale total score and its items suggesting more time connecting with friends and trying to connect with old friends;4) positive correlations with the Working Scale total score and its items including cooking, housekeeping, and creative work; 5) negative correlations with Stress Scale items suggesting feeling less isolated and lonely and less snacking;6) a negative correlation with the PROMIS Depression Subscale item on feeling hopeless; 7) negative correlations for the PROMIS Fatigue Subscale total score and all its items including feeling less fatigue, being less tired, and feeling less rundown; 8) negative correlations for the PROMIS Sleep Disturbance Subscale items including better sleep quality and more refreshing sleep; and 9) a negative correlation for the Posttraumatic Inventory-8 item indicating less feeling on guard.

Table 4. Correlation coefficients for significant relationships between touching self and scores on COVID-19 Lockdown Activities Survey scales and subscales.

\begin{tabular}{lcc}
\hline Measure & Correlation coefficient & plevel \\
\hline Health Scale Score & .50 & .001 \\
Connecting Scale Score & .14 & .05 \\
Working Scale Score & .17 & .01 \\
PROMIS Fatigue Subscale Score & -.23 & .01 \\
PROMIS Sleep Disturbances Score & -.15 & .05 \\
\hline
\end{tabular}

\section{Discussion}

Feeling touch deprived in the current study was experienced by $60 \%$ of the participants and correlated with living alone, but only $23 \%$ were living alone. This finding suggests that many who lived with others were also feeling touch deprived. Touch deprivation was negatively correlated with health behaviors which was not surprising given that touching your kids and your partner were items on the health scale and touching was the second most significant component of the factor analysis on the health scale. Physical health, although not measured here, would also be compromised by touch deprivation, as it would be expected to increase stress hormones and compromise immune function. Several studies have suggested that touch (moving the skin) stimulates pressure receptors under the skin which, in turn, leads to increased vagal activity, reduced stress hormones (e.g. cortisol) and increased natural killer cells that ward off bacterial and viral cells. ${ }^{10}$ These data highlight the need for stimulating pressure receptors via moving the skin, as in touching kids, touching partners/friends and selftouch.

Finding studies on touch deprivation in the literature was difficult, as already mentioned, given that touch deprivation effects have been almost exclusively limited to extreme touch deprivation situations. An exception was the absence of touching at airport gates among individuals traveling with each other, suggesting that touch deprivation was occurring before the COVID-19 lockdown. ${ }^{12}$

Literature has also been lacking on relationships between touch deprivation and negative mood states. Those that were correlated with touch 
deprivation in this study included stress, anxiety, and depression, as well as fatigue, sleep disturbances, and posttraumatic stress symptoms. These problems had been frequently reported in several studies on COVID-19 lockdowns..$^{1-6}$ Surprisingly, touch deprivation was not significantly correlated with worries about finances, although it was correlated with worries about getting the virus.

Finding literature on the positive effects of touching has also been difficult given that touch stimulation effects have been researched primarily as massage therapy effects on chronic psychiatric and medical conditions. ${ }^{10}$ Touching your kids and touching partners in the current study shared similar effects. First, they were both, not surprisingly, correlated with each other and associated with less touch deprivation and more exercise with others. Secondly, they both involved similar types of touching including hugging, kissing, backrubs, and cuddling. Thirdly, those who experienced more touching your kids and partners had similar demographics including being a younger age and living with others. They were also both correlated with higher scores on the health scale and higher scores on the working scale including engaging in more homework, caregiving, and housework.

Touching partners was also correlated with selftouch, and both in turn were associated with higher health scores. These results were not surprising given that touching others and selftouch have had similar effects including increased vagal activity, decreased stress hormones, and enhanced immune function. ${ }^{10}$ Touching partners was negatively correlated with stress, depression, and posttraumatic stress symptoms, suggesting that touching partners was more preventive of those lockdown problems. . $^{1,2,6}$

Self-touch, on the other hand, appeared to be more preventive of fatigue and sleep disturbances based on their negative correlations. $^{3-5}$ This is not surprising given that the most frequent self-touch activities in this survey, i.e. stretching, brushing in the shower, and yoga, have been defined as self-touch activities and have been noted to reduce sleep disturbances in other studies. ${ }^{16}$ Self-touch was also related to greater inside exercise, outside exercise, and exercise with others, suggesting that those who engaged in self-touch also engaged in all types of exercise. Pre-COVID research has also shown positive effects of exercise on anxiety ${ }^{17}$ and sleep disturbances. ${ }^{18}$ Exercise, touching your kids, and touching partners, as in hugging and back rubbing, would be expected to enhance immune function via moving the skin and stimulating pressure receptors under the skin, in turn, increasing vagal activity, decreasing cortisol and increasing natural killer cell activity to ward off viral and bacterial cells. ${ }^{8,17}$

Methodological limitations of these data include sampling and assessment issues. The sample was predominantly non-Hispanic, white women which would not be generalizable to the larger population. And, the self-report data are subject to questionable bias and reliability. Also, causality or direction effects could not be determined because the data are crosssectional rather than longitudinal. Unfortunately, because lockdowns like those of COVID-19 were not expected, no baseline data were available, as is the case for most of the COVID19 lockdown literature. Even without anticipating this lockdown, data on touch deprivation, touching your kids, touching partners, and selftouching would not have been available in the pre-COVID literature.

Despite these methodological limitations, these survey data analyses suggest that touch deprivation was associated with other COVID-19 lockdown problems including stress, depression, anxiety, fatigue, and PTSD symptoms. And touch deprivation and these lockdownassociated problems were reduced by touching partners and touching self. Further, all forms of touching including touching your kids, touching partners, and self-touching contributed to more health behaviors like exercise and home projects like cooking, housework, and creative 
projects. These may have confounded and compounded the positive effects of touching. Nonetheless, these data highlight the negative effects of touch deprivation and the positive effects of touching your yourkids, touching partners, and self-touching during lockdowns like those of COVID-19.

\section{References}

1. Stanton, R., To, Q., Khalesi, S., Williams, S., Alley, S., Thwaite, T., Fenning, A. \& Vandelanotte, C. Depression, Anxiety and Stress during COVID-19: Associations with Changes in Physical Activity, Sleep, Tobacco and Alcohol Use in Australian Adults International Journal of Environmental Research and Public Health. 2020: 17, 4065 DOI 10.3390/ijerph17114065

2. Field, T., Mines, S., Poling, S., Bendell, D. \& Veazey, C. Anxiety and depression during a COVID-19 lockdown.2020. Journal of Anxiety and Depression. 3(2), 124-137.

3. Huang, Y. \& Zhao, N. Generalized anxiety disorder, depressive symptoms and sleep quality during COVID-19 outbreak in China: A web-based cross-sectional survey. 2020. Psychiatry Research, 288, 112954.

4. Cellini, N., Canale, N. Mioni, G., \& Costa, S. Changes in sleep pattern, sense of time and digital media use during COVID-19 lockdown in Italy, Journal of Sleep Research. 2020, doi. org/10.1111/jsr.13074

5. Field, T., Mines, S., Poling, S., Bendell, D. \& Veazey, C. Stress and sleep disturbances during a COVID-19 lockdown. 2020. Journal of Psychology \& Mental Health.

6. Mines, S., Veazey C., Poling, S., Field, T. \& Bendell, D. PTSD symptoms and health behaviors. 2020. Annals of Psychiatry and Mental Health. 8(3), 1155-59.

7. Field, T. (1999). American Adolescents Touch Each Other Less and Are More Aggressive Toward Their Peers as Compared With French Adolescents. Adolescence, 34, 753-758.

8. Field, T. Social touch, CT touch and massage therapy. Developmental Review. 2019:51:123145.

9. Grewen, K. M., Anderson, B. J., Girdler, S. S., \& Light, K. C. Nonverbal encouragement of participation in a course: The effect of touching. Social Psychology of Education, 2003: 7: 89-98.

10. Field, T. Touch for socioemotional and physical well-being: A review. Developmental Review, 2010: 30: 367-383.
11. Cohen, S., Janicki-Deverts, D., Turner, R.B., \& Doyle, W.J. Does hugging provide stressbuffering social support? A study of susceptibility to upper respiratory infection and illness. Psychological Science, 2015: 26:135-147.

12. Field, T., Mines, S. \& Poling, S. Cell phones dominate waiting activity at airport gates. 2020. In preparation.

13. Field, T., Poling, S., Mines, S., Bendell, D. \& Veazey, C. Touch deprivation and exercise during a COVID-19 lockdown.2020. Medical Research Archives. 8(8), 1-12.

14. Dewitt, B., Feeny, D., Fischhoff, B., Celia, D., Hays, R.D. et al. Estimation of a preferencebased summary score for the patient reported outcomes measurement information system: The PROMIS-preference (PROPr) scoring system. Medical Decision Making. 2018: 38: 683-698.

15. Hansen, M., Anderson, T. E., Armour, C., Elklit, A., Palic, S., \& Mackrill, T. PTSD-8: A short PTSD inventory. Clinical Practice \& Epidemiology in Mental Health. 2010:6:101- 108.

16. Field, T. (2020). Yoga therapy research: A narrative review. Journal of Yoga and Physiotherapy. 8, 49-71.

17. Kandola, A. Vancampfort, D., Herring, M., Rebar, A. Hallgren. M. Firth, J. \& Stubbs, B. Moving to beat anxiety: epidemiology and therapeutic issues with physical activity for anxiety. Current Psychiatry Reports.2018; 20: 63 doi.org/10.1007/s11920-018-0923-x 